\title{
Alternative vs Polar Questions: the cornering effect
}

\author{
María Biezma \\ UMass Amherst
}

\section{Introduction}

The questions Are you making pasta? and Are you making pasta or not? can both be answered with yes or no. The semantics traditionally given to these questions does not predict any difference between the two, and yet, as illustrated in (1), the two questions are not interchangeable (see Bolinger 1978).
a. Would you marry me?
[POLQ]
b. Would you marry me or not?
[ALTQVN]

(1a) is perfectly fine as an initial marriage proposal. However, (1b) is perceived as odd in such a situation. The default interpretation of (1a) is that of a polar question (POLQ), whereas the default interpretation of (1b) is that of an alternative question in which two opposite alternatives are expressed (ALTQVN).

The question addressed in this paper is whether the difference between the sentences in (1) is due to a component that is part of the conventional linguistic meaning or attributable to pragmatic inferences. The answer offered in this paper is yes, in both cases. I argue that intonation is crucial for the semantics of questions like (1), leading to a difference in the interpretation of the possible answers. This difference in the semantics shapes discourse structure and accounts for pragmatic effects.

The paper is organized as follows: in $\S 2$ I illustrate the differences between the two kinds of questions by reviewing the data offered in Bolinger (1978). I offer new data illustrating what I call the cornering effect. I argue that this effect is at the core of the difference between PoLQs and AlTQVNs, and look for an account in the rest of the paper. In $\S 3$ I review the semantics of POLQs and ALTQvNs, taking into account the meaning effects of their intonation. In order to do this I make use of Zimmermann's (2000) insights on the semantics of lists and the role of intonation. In $\S 4$ I make use of Roberts' (1996) and Büring's (2003) theories of discourse to explain the different pragmatic effects of POLQs and ALTQVNs in view of their different semantics. In $\S 5$ I go back to Bolinger's data and show how the proposal offered in this paper accounts for it.

I am extremely thankful to Rajesh Bhatt, Lyn Frazier and Christopher Potts for their comments and help. Also, many thanks to the Semantics Reading Group at UMass Amherst, specially to Annahita Farudi. Of course, all errors are my own. 


\section{The problem}

Both questions in (2) can be answered with yes or no.
a. Are you making pasta?
[POLQ]
b. Are you making pasta or not?
[ALTQvN]

However, the two questions are not interchangeable. Bolinger (1978) points out different scenarios in which POLQs are acceptable and ALTQVNs are not. In requests (3a), inference drawing (3b), rhetorical questions (3c), invitations (2d) and conversation starters (3e) it is only appropriate to use a POLQ.

PolQs Vs AltQvNs:

a. Requests: Marriage proposal

i. Will you marry me?

ii. Will you marry me or not? odd [ALTQVN]

b. Drawing inferences: A conversation talking about David

i. A: I just saw David

B: Is David back from Toronto?

ii. B': Is David back from Toronto or not? odd [ALTQVN]

c. Rhetorical Questions: Your friend is telling you what he did last night

i. Are you crazy?

ii. Are you crazy or not? odd [ALTQVN]

d. Invitations: Your friends just arrived at your house

i. Do you want something to drink?

ii. Do you want something to drink or not? odd [ALTQVN]

e. Conversation Starters: Trying to start a casual conversation

i. Do you like to play golf?

ii. Do you like to play golf or not? odd [ALTQVN]

In addition to the differences between POLQs and AltQvNs noted by Bolinger, (3), there is a difference between the two types of questions in terms of the options made available to the addressee. I will term this the cornering effect. It is illustrated in (4). ${ }^{1}$

(4) Scenario: You are in charge of coordinating the cooks for the colloquium dinner. John is one of the cooks. You talked to John yesterday and he said he would make stew but did not confirm whether he would also make pasta. Dinner is tomorrow and you need to know what is happening with the pasta.

\footnotetext{
${ }^{1}$ van Rooy and Safárová (2003) have recently provided a purely pragmatic account of POLQs vs. ALTQVNs according to which POLQs are special because their positive answer has a higher utility value for the questioner. I cannot discuss their proposal here for reasons of space. However, they are not concerned with data exemplifying the cornering effect.
} 
a. You: Are you making pasta?

John: (Silence and dubitative faces)

You: Are you making pasta or not?

b. You: Are you making pasta?

John: (Silence and dubitative faces)

You: (C'mon) Are you making pasta?

Intuitively, when an ALTQVN is used, (4a), there is a reduction in the set of responses available to the addressee. As (4a) illustrates, the questioner uses an ALTQVN to try to 'force' an answer from the addressee. The use of a question asking about logically opposite alternatives, you make pasta, $p$, and you don't make pasta, $\neg p$, results in the cornering of the addressee. This effect is not observed if the POLQ is instead uttered again. As (4b) illustrates, the effect, if any, is that of insistence.

The cornering effect described above indicates that ALTQVNs are the preferred questions for seeking information when the addressee appears to be withholding it and the speaker wishes to close the issue. Empirical support for this observation was obtained with a questionnaire experiment that tested speaker's intuitions regarding questions. In the experiment, participants were presented with scenarios like (4) and were asked which question they would choose if they wanted to obtain the relevant information and end the conversation. An example of the materials is presented in (5).

(5) You are hosting a party and offering drinks to everybody. You have asked your close friend Loren whether she wants a beer, and she has been talking to you for 10 minutes about how badly she wants a beer and worrying about how many calories they have. You want to move on and find out whether Loren does actually want a beer. Which of the next questions would you ask in order to conclude the conversation and find out about the beer?

\begin{tabular}{|l|l|}
\hline a. Do you want a beer? & \\
\hline b. Do you want a beer or not? & \\
\hline
\end{tabular}

10 different scenarios like (5) were presented to 36 UMass Undergraduate students with no linguistic training. The results indicated that, in such scenarios, $65.4 \%$ of the responses favored an ALTQvN over a POLQ. There was a significant difference: $\mathrm{p}<0.05 .^{2}$ The questionnaire results confirmed the intuitive descriptions of the cornering effect.

The interpretation traditionally given to POLQs and AltQvNs does not capture the differences illustrated in (3) and (4a). If we consider the question operators proposed by Hamblin's (1973) and Karttunen's (1977), (6), the semantics of the sentences in (3) are similar. The question operators scoping over the denotation of you make pasta and you make pasta or not induce similar partitions (Romero and Han 2003).

\footnotetext{
${ }^{2}$ The experiment was controlled with items in which the scenarios involved starting a conversation. In those cases, participants preferred a PoLQover an ALTQVN $(\mathrm{p}<0.05)$.
} 
Questions operators

a. $\llbracket Q_{\text {yes/no }} \rrbracket=\lambda p_{\langle\mathrm{s}, \mathrm{t}\rangle} \cdot \lambda w_{\mathrm{s}} \cdot \lambda q_{\langle\mathrm{s}, \mathrm{t}\rangle} \cdot[q=p \vee q=\neg p]$

b. $\llbracket Q_{\mathrm{Alt}} \rrbracket=\lambda p_{\langle\mathrm{s}, \mathrm{t}\rangle} \cdot \lambda w_{\mathrm{s}} \cdot \lambda q_{\langle\mathrm{s}, \mathrm{t}\rangle} \cdot p=q$

When $\llbracket Q_{\text {yes/no }} \rrbracket$ scopes over the denotation of you make pasta, it induces a partition of the set of possible worlds into a set in which you make pasta and a set in which you do not make pasta. The same is true when $\llbracket Q_{\text {Alt }} \rrbracket$ scopes over the denotation of you are making pasta or not.

a. Polar questions: Are you making pasta?

$$
\begin{aligned}
\llbracket(2 \mathrm{a}) \rrbracket\left(w_{0}\right)=\quad \lambda q[q & =\lambda w \cdot \operatorname{make}(\text { pasta }, \text { you }, w) \\
& \vee q=\lambda w . \neg \operatorname{make}(\text { pasta }, \text { you }, w)]
\end{aligned}
$$

b. Alternative Questions: Are you making pasta or not?

$$
\begin{aligned}
\llbracket(2 \mathrm{~b}) \rrbracket\left(w_{\mathrm{0}}\right)=\lambda q[q= & (\lambda w \cdot \operatorname{make}(\text { pasta }, \text { you }, w) \\
& \vee \lambda w . \neg \operatorname{make}(\text { pasta }, \text { you }, w))]
\end{aligned}
$$

In this paper I argue that the semantics of (2a) and (2b) are actually different. The difference in the semantics leads to a difference in the pragmatics that explains the cornering effect and also the data in Bolinger (1978).

\section{The semantics: exhaustive alternatives}

In this section we will see that questions expressing alternatives have two main intonational patterns. They may have a final rising or falling intonation. I illustrate the effects of these two patterns in discourse and link the differences obtained from these two possible intonations in questions to the phenomenon observed in lists (Zimmermann 2000). We can derive the relevant differences between POLQs and AltQvNs with a semantics that characterizes both POLQs and ALTQvNs as involving lists of alternatives (spelled out or not): POLQs involve an open list (with just one alternative spelled out), indicated by final rising intonation, ${ }^{3}$ whereas $\mathrm{AL}$ TQVNs involve closed lists (with all alternatives spelled out), indicated by final falling intonation. Open and closed lists differ semantically with respect to the absence/presence of a Closure operator acting on the possible alternatives (Zimmermann 2000). I treat the information provided by the closure operator as a presupposition.

\footnotetext{
${ }^{3}$ Notice that the term POLQ is only used for questions that follow this pattern (they spell out one alternative and have final rising intonation).
} 


\subsection{A difference in intonation: Yes/No vs Alternative readings}

ALTQVNs are a particular case of questions expressing alternatives. (8) is an example of an alternative question that is not an ALTQvN.

a. Are you making pasta or fish?

b. Are you making pasta or making fish $?^{4}$

The default reading for questions like (8a) is the alternative reading: Which of these two things are you doing: making pasta or making fish? However, questions presenting alternatives have another reading, a yes/no reading: is it the case that you are making pasta or fish? Pruitt $(2007,2008)$ shows that the yes/no reading is not the default reading. It appears when the question is uttered with its non-default intonation. The two intonations associated to the two possible readings are specified in (9). ${ }^{5}$

\section{Fall Vs Rise}

a. Are you making pasta L $^{\star H}$ - or fish H'LL\% ? [Final Fall=Alternative] Which of these things are you doing: making pasta or making fish?

b. Are you making pasta or fish $\mathbf{L}^{\prime} \mathbf{H} \cdot \mathbf{H} \%$ ?

[Final Rise $=\underline{\mathrm{Yes}} / \mathrm{No}]$

Is it the case that you are making pasta or fish?

As Pruitt shows, the crucial difference that leads the human language processor to interpret the question as either an alternative question or a yes/no question is the final intonation. The alternative reading comes about when the final intonation is falling, whereas the yes/no reading comes about when there is final rising intonation.

(10) Scenario: You are in charge of coordinating the cooks for the colloquium dinner. John one of the cooks. The menu is pasta, fish and stew.

a. (You have already assigned the task of making stew to someone else.)

You: Are you making pasta $\mathrm{L}^{*} \mathrm{H}-$ or fish $_{\mathrm{H}^{*} \mathrm{~L}-\mathrm{L} \%}$ ?

John: (No) I am making stew. [odd]

John': Wait, I wanted to make stew, isn't that possible?

\footnotetext{
${ }^{4}$ I follow Romero and Han (2003) in assuming ellipsis in the second disjunct.

${ }^{5}$ I follow Pruitt (2007) in characterizing the intonation of alternative questions and yes/no questions. According to Pruitt, "a canonical alternative contains (i) a pitch accent on the first disjunct; (ii) a $\mathrm{H}$ phrasal/boundary tone at the end of the first disjunct; (iii) a $\mathrm{H}$ pitch accent on the final disjunct; and (iv) L phrasal and boundary tones (L-L\%) on the final disjunct. Features (i) and (ii) create the impression of a rise at the end of the first disjunct, while (iii) and (iv) cause the perception of a final fall. Features (i) and (ii) are often accompanied by a pause before or, but this does not appear to be required. Some have also noted that there is variation in the identity of the pitch accents in an Alt question (whether $\mathrm{H}$ or L), and perhaps in the phrasal tone of the non-final disjunct (again, whether H or L)." With respect to yes/no questions Pruitt (2007) indicates that "the notable features of a yes/no question, when defined in parallel to an alternative question are: (i) no pitch accent on the first disjunct; (ii) no phrasal/boundary tone on the first disjunct; (iii) a $\mathrm{L}$ pitch accent on the final disjunct; and (iv) $\mathrm{H}$ phrasal and boundary tones $(\mathrm{H}-\mathrm{H} \%)$ on the final disjunct. The combination of features (iii) and (iv) in yes/no questions creates the percept of a final rise, while a lack of a significant pitch movement is characteristic of the first disjunct."
} 
b. (All the options are available.)

You: Are you making pasta $\mathrm{L}^{*} \mathrm{H}-$ or $\mathrm{fish}_{\mathrm{L}^{*} \mathrm{H}-\mathrm{H} \%}$ ?

John: (No) I am making stew. $\sqrt{ }$

The utterance of (10a) encodes that the only possibilities for John are either making pasta or making fish. By uttering (10a), the speaker limits the possible answers to those alternatives specifically expressed in the question. At this point in the discourse, it is understood by both participants in the conversation that no other alternative is available. To invoke a different alternative is not discursively appropriate. The speaker presupposition is indicated by the final falling intonation, and it needs to be accommodated by the addressee. This is illustrated by John's alternative response (perfectly fine), in which he wonders what happened to the possibility of making stew. John is wondering why the questioner is assuming that stew is not an option anymore.

Things are different in the case of (10b). The question in (10b) has a yes/no reading (notice the final rise): is it the case that you are making pasta or fish? John's response in this case is not odd, since the questioner is not indicating that (s)he assumes that making pasta or making fish are the only alternatives available. ${ }^{6}$ The questioner may have guessed that pasta or fish are amongst John's favorite dishes and decided to spell them out. However, the questioner is not ruling out the possibility that other alternatives are in the picture.

As interim summary, we have seen that questions spelling out two alternatives have at least two possible intonational patterns, identifying two different readings. With default intonation (final fall), the alternative reading is obtained. Responses to these questions that are not formulated in terms of the explicit alternatives are odd. When the intonation has a final rise (and no phrasal/boundary tone in the first disjunct) the yes/no reading is obtained. Responses to these questions are fine even if they refer to alternatives that are not spelled out. For this reason, John's appeal to stew does not sound odd in (10a).

ALTQvNa are simply alternative questions that spell out opposite alternatives, $p$ and $\neg p$.

Are you making pasta $\mathrm{L}^{*} \mathrm{H}-$ or $\operatorname{not}_{\mathrm{H}^{*} \mathrm{~L}-\mathrm{L} \%}$ ?

Contrary to the case of regular alternative questions, ALTQVNs have only one possible intonation: final falling intonation. In what follows I argue that final falling intonation indicates exhaustivity. Questions asking about opposites naturally exhaustify the possible answers. Falling intonation in ALTQvNs signals that the alternatives spelled out are exhaustive.

Let us now turn to questions in which only one alternative is spelled out and we have final rising intonation, (12).

$$
\text { Are you making pasta } \mathbf{L}^{*} \mathbf{H}-\mathbf{H} \% ? \quad[\text { Final rise }=\underline{\text { yes/no }}] \quad(\text { POLQ })
$$

\footnotetext{
${ }^{6}$ Notice that John's response I am making stew in (10b) is indeed an answer: via pragmatic enrichment we understand that the answer to the quesition is "no", and John supplies further information, namely, that he is actually making stew.
} 
Exactly like in the case in which two alternatives are spelled out, with final rising intonation we have (again) a yes/no reading. This is the default intonation. Responses that make reference to alternatives that are not spelled out are acceptable in the discourse (this case is similar to the case of two alternatives spelled out).

(13) Scenario: You are in charge of coordinating the cooks for the colloquium dinner. John is one of the cooks.

a. You: Are you making pasta $_{\mathbf{L}^{*} \mathbf{H}-\mathbf{H} \%}$ ?

John: (No) I am making stew.

Via pragmatic enrichment we infer that the answer to the question is "no" with regard to pasta, since he is actually making stew. The summary so far is in Figure 1.

\begin{tabular}{|c||l|l|c|}
\hline \# Alternatives & Final intonations & Reading & Exhaustivity \\
\hline \hline \multirow{2}{*}{ 2 alternatives spelled out } & final fall & alternative reading & $\sqrt{ }$ \\
\cline { 2 - 4 } & final rise & yes/no reading & $\mathrm{X}$ \\
\hline \hline 1 alternative spelled out & final rise & yes/no reading & $\mathrm{X}$ \\
\hline
\end{tabular}

Figure 1: Alternatives/intonation/reading correspondence

Questions with only one alternative spelled out and final falling intonation are also possible, (14), although they are dispreferred.

(14) Are you making pasta ${ }_{\mathbf{H}^{*} \mathrm{~L}-\mathrm{L} \%}$ ?

It is difficult to see what a question like (14) means: it asks about only one alternative but it also claims that it is the only possibility available. It cannot be interpreted as a yes/no question. ${ }^{7}$ Questions like (14) behave like AltQvNs, (15).

(15) Scenario: You are in charge of coordinating the cooks for the colloquium dinner. John is one of the cooks. You talked to John yesterday and he said he would make stew but did not confirm whether he was also going to make pasta. The dinner is tomorrow and you need to know what is happening with the pasta.

a. You: Are you making pasta $\operatorname{H}^{*} \mathbf{L}-\mathbf{L} \%$ ?

John: I am making stew.

[odd]

You: I know that, but what about pasta? Are you making pasta $\mathbf{H}^{*} \mathrm{~L}-\mathrm{L} \%$ ?

John's answer in (15) is odd because it refers to an alternative that the questioner is not considering available at the time of utterance, as indicated by the final falling intonation. John would only respond in this way if he wanted to be defiant and end the conversation by stating that he does not care about what you consider to be the possibilities available. He is going to make stew no matter what. As in (10a), the addressee would be aware that he is somehow defying the assumptions regarding exhaustivity being made by the questioner.

\footnotetext{
${ }^{7} \mathrm{~A} \llbracket Q_{\text {yes/no }} \rrbracket$ operator like (6a) cannot scope over TP in this case because exhaustivity (signaled by final falling intonation) indicates that the only possible alternative is you make pasta, p. A $\llbracket Q_{\text {yes/no }} \rrbracket$ scoping over it would also consider the negated alternative, $\neg p$, incurring in a contradiction. In general, this is true for every case in which exhaustivity is signaled.
} 
To summarize, in this section we have seen that differences in the final intonation in questions presenting alternatives lead to differences in discourse. When the final intonation is falling, the speaker signals to the addressee that (s)he assumes that the alternatives spelled out are the only alternatives available in the discourse. When the final intonation is rising, the speaker indicates that (s)he considers that the alternatives spelled out in the question are not the only contextually available ones.

In the next section I incorporate the effects of intonation in the semantics of questions to account for the data illustrated in this section. In order to do so, I make use of Zimmermann's (2000) proposal for lists.

\subsection{The link with open and closed lists}

Zimmermann establishes a link between intonation and lists of assertive disjunctions, illustrated in the example in (16).

(Q) Which tube stations are one stop from Oxford Circus?

(L) Piccadilly Circus, Bond Street, Tottenham Court Road, Green Park, Warren Street, Regent's Park [are each one stop from Oxford Circus] $]_{\mathbf{L}^{*} \mathbf{H}-\mathbf{H} \%}$ (C) ... and no other underground station [is one stop from Oxford Circus].

[Zimmermann 2000: ex. (12)]

Imagine someone asks the question in (16Q), and the answer lists possible stops one stop from Oxford Circus, followed by the remark in (16C), signaling that the previous list is exhaustive. Notice that the list's final intonation in this situation is rising. Imagine now that there is no (16C) indicating exhaustivity, (17).

$$
\begin{aligned}
& \text { (L) ..., Green Park, Warren Street, Regent's Park } \mathbf{L}^{*} \mathbf{H}-\mathbf{H} \% \\
& \text { (C) (silence) }
\end{aligned}
$$

As Zimmermann points out, if the list is not closed explicitly and the numeration of stations finishes with a final rise, the addressee is left with the impression that the list is not exhaustive. However, if the intonation at the end of the numeration is falling instead of rising the addressee is left with a very different impression.

$$
\begin{aligned}
& \text { (L) ..., Green Park, Warren Street, Regent's Park } \text { H*L-L\% }^{*} \text { (C) (silence) }
\end{aligned}
$$

When the numeration of stations finishes up with falling intonation, even if there is no final remark stating that the list is exhaustive, the addressee understands that there are no other stations one station away from Oxford Circus. Zimmermann proposes that the difference between lists finishing with final falling intonation and lists with final rising intonation is the presence of a closure operator present in the semantics triggered by the final falling intonation.

The phenomenon illustrated above with disjunctive list of assertions is exactly the same phenomenon we saw in the previous section: when the final intonation is falling, questions spelling out alternatives indicate that the list of alternatives offered is exhaustive, whereas when the final intonation is rising, they indicate that 
there are more contextually available alternatives than those spelled out. In the next section I argue that POLQs, which have final rising intonation, do not have a closure operator in the semantics. This indicates that other alternatives, besides those mentioned, may be available. ALTQvNs, however, have final falling intonation, indicating that there are no more alternatives available in the discourse besides those offered.

\subsection{The semantic components}

In this section I present the ingredients of the anlysis: the denotations of sentences, the question operators, and the closure operator. In what follows I make use of a Hamblin-style semantics and characterize the denotation of a sentence as a set containing a proposition. I assume that there is no or in the semantics (Zimmermann 2000, Geurts 2005, Alonso-Ovalle 2006), and disjunction only presents lists. Sentences with or denote the union of the sets corresponding to the disjuncts (AlonsoOvalle 2006), (19).

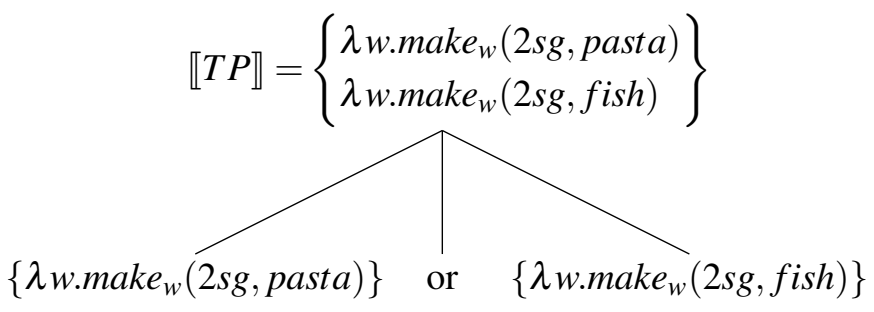

For practical reasons, I make use of an extensional version of the question operators we saw in (6), adapted to take scope over sets of propositions.

a. $\llbracket Q_{\text {yes/no }} \rrbracket=\lambda S_{\langle\langle s, t\rangle, t\rangle} \lambda q \cdot q=\left(\bigvee_{r \in S}\right) \vee q=\neg\left(\bigvee_{r \in S}\right)$

b. $\llbracket Q_{\mathrm{Alt}} \rrbracket=\lambda S_{\langle\langle s, t\rangle, t\rangle} \cdot \lambda q . q=r_{1} \vee \cdots \vee q=r_{|S|}, r_{i} \in S$ for all $1 \leq i \leq|S|$

The information provided by the closure operator is treated as a presupposition associated with closed-lists questions. ${ }^{8}$

$$
\Gamma:=\lambda G_{\langle\langle s, t\rangle, t\rangle}:(\forall q)[\text { EpistemicallyAvailable }(q) \leftrightarrow q \in G] . G
$$

When the operator in (22) applies to a set of propositions, G, it returns the same set of propositions and checks the presupposition that these propositions are the only propositions epistemically possible. In that sense, the list is closed: the only epistemically possible alternatives are the spelled out alternatives. As in Zimmermann (2000), the presence of closure in the semantics is indicated by final falling intonation. ${ }^{9}$

\footnotetext{
${ }^{8}$ The operator in (22) is inspired in Zimmermann's closure operator, (21).

(21) $\Gamma_{P}:=[\lambda G(\forall x)(\forall q)[[q=P(x) \& \operatorname{True}(q)] \rightarrow q \in G]]$

where $\mathrm{G}$ ranges over groups of propositions and ' $\in$ ' denotes group membership

Notice that Zimmermann considers sets of propositions that are modalized. The reader is referred to Zimmermann (2000) for details. The proposal has been modified to allow the operator to act over sets of propositions.

${ }^{9}$ Notice that the operator in (22) is not completely right. Obviously, there are many independent propositions that are also epistemically available, such as it is raining right now. What we want
} 
With these ingredients in place, let us start by examining the resulting semantics of questions with final falling intonation. These are the questions in which $\Gamma$ is present in the semantics.

a. Are you making pasta ${ }_{\mathrm{L}^{*} \mathrm{H}-}$ or fish $_{\mathbf{H}^{*} \mathbf{L}-\mathbf{L} \%}$ ?

b.

$$
\lambda q \cdot q=\lambda w . \text { make }_{w}(2 s g, \text { pasta }) \vee q=\lambda w . \text { make }_{w}(2 s g, f i s h)
$$

(Presupposition: the only epistemic alternatives are make(2sg,pasta) and make(2sg,fish))

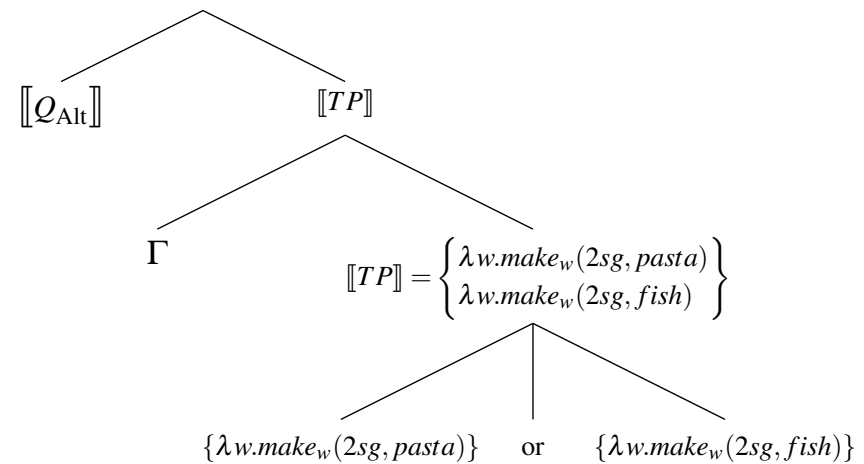

a. Are you making pasta $\mathrm{L}^{*} \mathrm{H}-$ or $\operatorname{not}_{\mathbf{H}^{*} \mathrm{~L}-\mathrm{L} \%}$ ?

b.

$$
\lambda q . q=\lambda \text { w.make }_{w}(2 s g, \text { pasta }) \vee q=\lambda w . \neg \text { make }_{w}(2 s g, \text { pasta })
$$

(Presupposition: the only epistemic alternatives are make(2sg,pasta) and $\neg$ make(2sg,pasta))

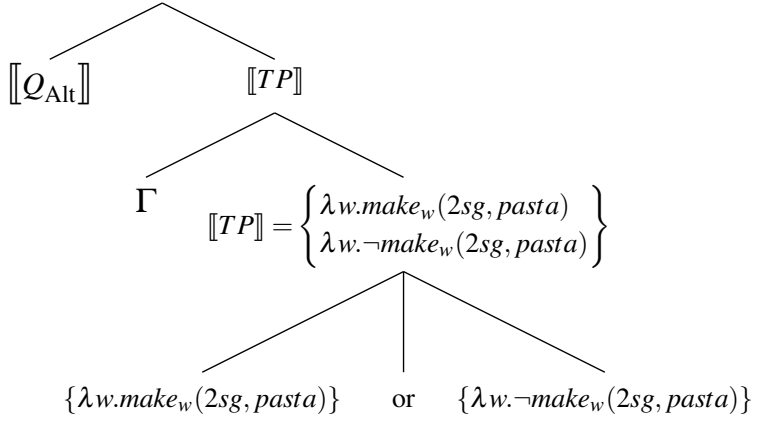

Both in the case of regular alternative questions, (23), and AltQvNs, (24b), there is a closure operator in the semantics indicated by the final falling intonation. The final denotation encodes the presupposition that the only available alternatives are those asked about in the question. Let us turn now to questions with final rising intonation.

\section{a. Are you making pasta $_{\mathbf{L}^{*} \mathbf{H}-\mathbf{H} \%}$ ?}

b.

$$
\lambda q \cdot\left[q=\lambda \text { w.make }_{w}(2 s g, \text { pasta }) \vee q=\lambda w . \neg \text { make }_{w}(2 s g, \text { pasta })\right]
$$

closure to introduce in the denotation of a question like are you making pasta $_{L^{*} H-}$ or fish ${ }_{H^{*} L-L \%^{2}}$ ? is the presupposition that for all the things you could make, the only ones epistemically possible are that you make either pasta or fish. One could solve this problem by making reference to the contextually salient epistemic alternatives. I do not have space to introduce the mechanisms that would solve the problem, nor the different theoretical challenges they present, thus I leave this question open. Also, one may want to consider the discussion introduced in Geurts (2005), who claims that the relevant notion of possibility is not purely epistemic, and may also be deontic. 
a. Are you making pasta or fish $_{\mathbf{L}^{*} \mathbf{H}-\mathbf{H} \%}$ ?

b.

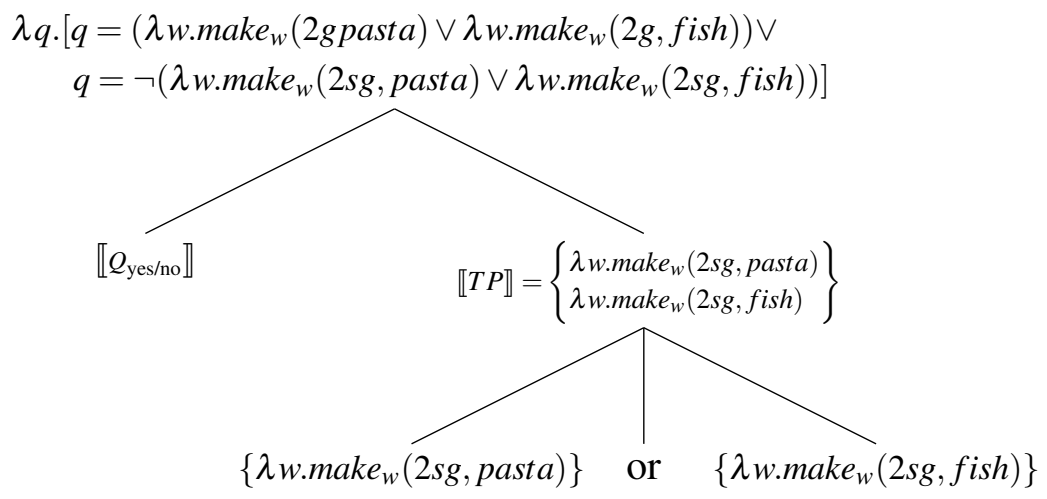

When the final intonation is rising, there is no closure operator and thus there is no presupposition regarding the exhaustivity of the alternatives, (25-26).

To summarize, I have claimed that both POLQs and ALTQVNs involve lists. In the case of POLQs, these are open lists, indicated by the final rising intonation, whereas in the case of ALTQVNs the lists are closed, as indicated by the final falling intonation. The difference in intonation signals a difference in the semantics, i.e., the absence/presence of a closure operator carrying presuppositional information, (27).

a. $\llbracket$ Are you making pasta $\mathrm{L}_{\mathrm{L}^{*} \mathrm{H}-}$ or $\operatorname{not}_{\mathrm{H}^{*} \mathrm{~L}-\mathrm{L} \%} ? \rrbracket=$ $\lambda q . q=\lambda$. $_{\text {make }}(2 s g$, pasta $) \vee q=\lambda w . \neg$ make $_{w}(2 s g$, pasta $) \quad$ [ALTQVN] Presup: the only epistemic alternatives are make(2sg,pasta) $\& \neg$ make(2sg,pasta)

b. «Are you making pasta $\mathbf{L}_{\mathbf{L}} \mathbf{H}-\mathbf{H} \% ? \rrbracket=$

$\lambda q . q=\lambda$ w.make $_{w}(2 s g$, pasta $) \vee q=\lambda w . \neg$ make $_{w}(2 s g$, pasta $) \quad$ [POLQ]

In what follows I introduce a hierarchical discourse model and illustrate how this difference in the semantics leads to differences in the discourse.

\section{The discourse: a cul de sac}

I argue that ALTQVNs are the last discourse move by the questioner: they cannot be followed by further questions. PoLQs, on the other hand, are not the last possible move. They give the addressee more room to maneuver.

\subsection{Background}

Bulding on Roberts (1996), Büring (2003) proposes a hierarchical discourse model that can be represented by d(iscourse)-trees. Like Roberts, Büring considers the discourse to be structured by (implicit) questions. Following Stalnaker (1978), Roberts states that "the primary goal of disourse is communal inquiry. [...] But we must develop strategies for achieving this goal, and these strategies involve subinquiries. As in a game, some strategies may be better, some worse." Questions, when accepted, drive the discourse following a strategy whose goal is to answer the 
Big Question (in Roberts' terms, i.e., the ultimate question we want to answer). ${ }^{10}$ Büring (2003) uses representations like the one in (28).

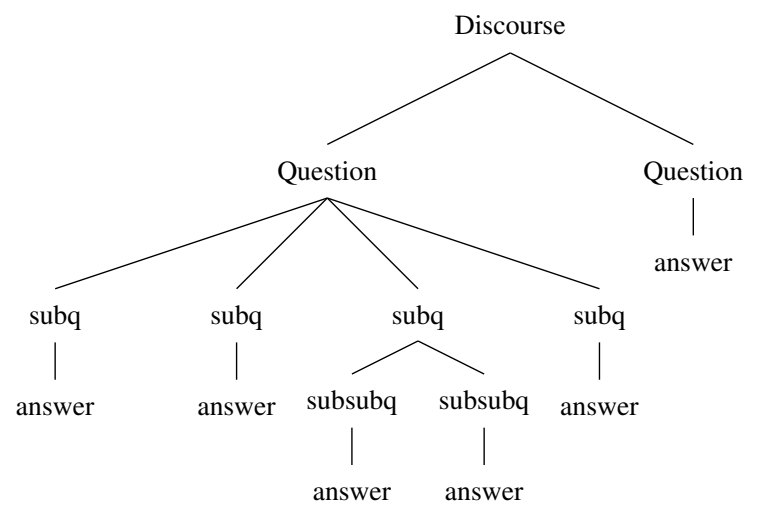

[Büring 2003: ex.(6)]

The discourse model proposed by Büring (2003) and illustrated in (28) is a hierarchical one. Every node is a move. In Büring's terms, a move is a syntactic phrase marker in the d-tree, and the d-trees encode a total ordering of moves. A questionanswer pair, (29a), is well-formed if and only if there is a d-tree which contains it. In the same way, the system predicts that "a question-question sequence is wellformed if there can be a d-tree in which Q1 immediately dominates Q2," (29b). ${ }^{11}$

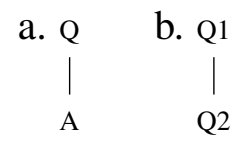

[Büring 2003: ex (7)]

Büring sketches two conditions that characterize the set of well-formed d-trees: informativity, (30a), and relevance, (30b).

(30) a. Informativity: Don't say known things, don't ask for known things!

b. Relevance: Stick to a question until it is sufficiently resolved!

[Büring 2003: ex (8)]

As Büring points out, informativity can be checked against the participants' common ground (Stalnaker 1978). With respect to relevance, Büring assumes that " $\mathrm{A}$ is an answer to $Q$ if A shifts the probabilistic weights among the propositions denoted by $Q$. ." Büring's first attempt to define relevance is in (31) (Büring 2003: ex. (9)).

Relevance

a. an assertion $\mathrm{A}$ is relevant in a d-tree DT iff $\mathrm{A}$ is an answer to the $\mathrm{Q}$ (uestion) $\mathrm{U}$ (nder) D(discussion) for A in DT. ${ }^{12}$

\footnotetext{
${ }^{10}$ This is the term that Roberts (1996) uses. Conceptually, it corresponds to the top-most node in Büring's tree (see below).

${ }^{11}$ See Büring (2003) for specific conditions on well-formed trees.

${ }^{12}$ For any move $\mathrm{M}$, the question under discussion is the move M? immediately dominating it. (Büring 2003: pg 7)
} 
b. a question Q is relevant in a d-tree DT iff at least one answer to Q is an answer to the QUD for Q in DT.

These constraints, as they stand in Büring (2003), simply sketch a theory of discourse. However, they will be of good service to us in what follows. In the next section we will see how a hierarchical discourse model like the one presented in this section, in combination with the semantics of PoLQs and AlTQvNs, can account for the pragmatic effects we have observed.

\subsection{The semantics and the discourse}

Let us start by looking at questions with rising intonation (corresponding to a lack of closure in the semantics). A d-tree for a PolQ like are you making pasta L $^{*} \boldsymbol{H}-\boldsymbol{H}_{\%}$ ? might look like (32).

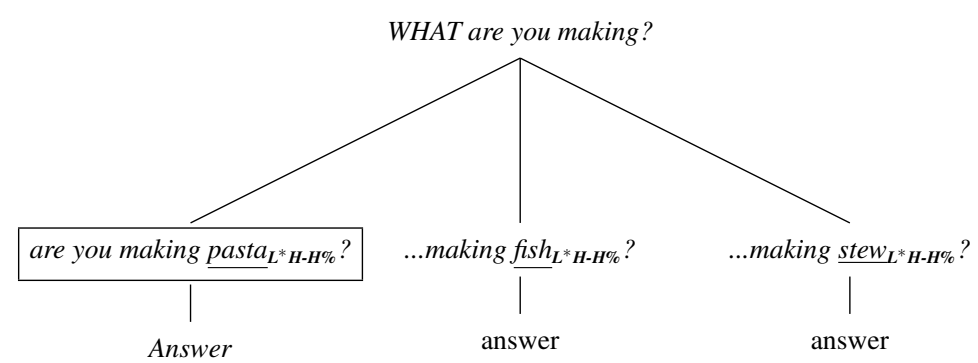

The d-tree in (32) is constructed taking into consideration the focus structure of the questions. The question are you making pasta? has the focus structure in (33b), indicated by its pitch accent, (33a). ${ }^{13}$

a. Are you making pasta $_{\mathbf{L}^{*} \mathbf{H}-\mathbf{H} \%}$ ?

b. Are you making [pasta $]_{F}$ ?

(33b) is a POLQ with final rising intonation, and involves an open list. Since the list is open, there are other epistemically possible/relevant alternatives which are not spelled out (Zimmermann 2000). I propose that the other members of the list (not spelled out) are the other sisters in the d-tree. Substituting the focused word (the only explicit member of the list) by other epistemically possible contextual alternatives, we obtain all the questions that are sisters of this question. All those questions are also sub-questions of the wh-question what are you making?, resulting from substituting the $w h$ - word by all the alternatives. The d-tree in (32) also illustrates the relationship between questions, an entailment relationship:

A question Q1 entails another question Q2 iff answering Q1 yields a complete answer to Q2. [Groenendijk and Stokhof 1984]

Any question on a d-tree entails any of its daughter-questions. For example, in (32), what are you making? entails any of its daughters, since a complete answer to it is an answer to any of its daughters. ${ }^{14}$

\footnotetext{
${ }^{13}$ Focus anywhere else would need an intonation different from the default intonation in (33a).

${ }^{14}$ The claims made about entailment here hold only if the semantics of wh-questions is exhaustive, as in Groenendijk and Stokhof (1984).
} 
The d-tree in (32) illustrates that POLQs can be used as strategies to answer higher questions: even though the addressee is asked about pasta in (33b), it is understood that the question under discussion is what are you making?, and all the sisters of the uttered question are open alternatives (i.e., remain as possible epistemic alternatives). This proposal predicts that the dialogue in (35) is fine.

A: Are you making pasta $_{\mathbf{L}^{*} \mathbf{H}-\mathbf{H} \%}$ ?

B: Actually, I would prefer to make stew.

The dialogue in (35) illustrates that B considers that there are other alternatives open to him besides those presented by A. In terms of the semantics of questions, Actually, I would prefer to make stew is an answer to the question are you making pasta?: via pragmatic enrichment we can conclude that the addressee is not making pasta, since he wants to make stew.

The case of questions with final rising intonation and overt disjunction is similar to POLQs (there is no closure operator and $\llbracket Q_{\text {yes/no }} \rrbracket$ takes scope over the set of propositions). The discourse situation is alike in that there are epistemically available alternatives other than the alternatives spelled out. In our system this means that the question spelled out has sisters: the other epistemically available alternatives. The difference between POLQs and questions expressing two alternatives with final rising intonation is the number of alternatives spelled out: in the case of POLQs there is only one alternative spelled out, whereas in the case of questions with overt disjunction and rising intonation there is more than one alternative spelled out.

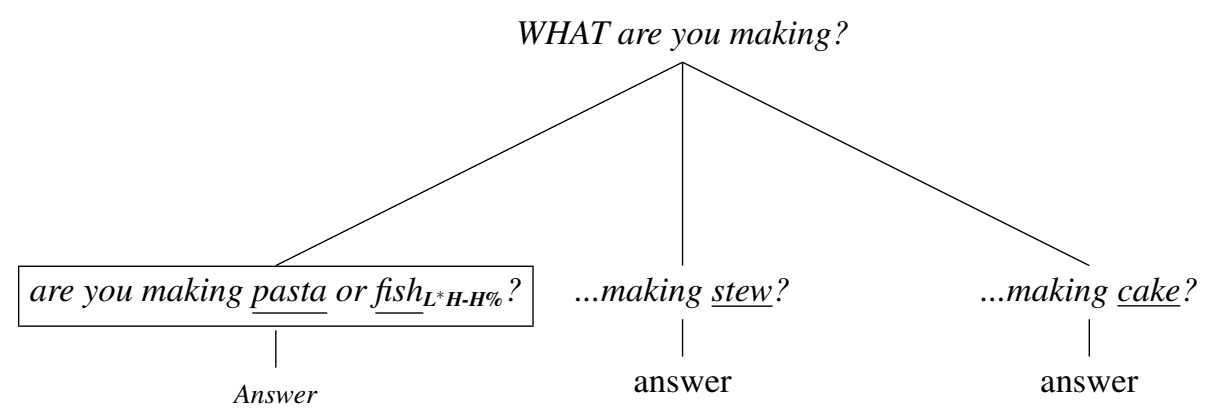

Comparing (32) and (36), we can see that the discourse situation at the time of utterance is the same in both cases.

Let's go a step further: what about sequences of questions in which an ALTQvN follows a PolQ? Taking into consideration the above discourse model, we should check whether the Q1-Q2 sequence in (37) is an appropriate discourse.

Are you making pasta?

Are you making pasta or not?

The moves in (37) are in an entailment relation, (34). Let us put the strategy in (37) in context, (38).

(38) Scenario: A wants to find out what B wants to cook for the colloq:

A: Are you making pasta? 


\section{B: (silence and dubitative faces) \\ A: Are you making pasta or not? \\ B: $\mathrm{No} / \mathrm{Yes}$}

Seeing that the addressee is not quite decided, the questioner tries to close the issue by following his previous question with an ALTQVN, i.e., using a substrategy. This dialogue could be part of a d-tree like (39). The effect of the ALTQVN is that of 'forcing' the addressee to give an answer with respect to pasta.

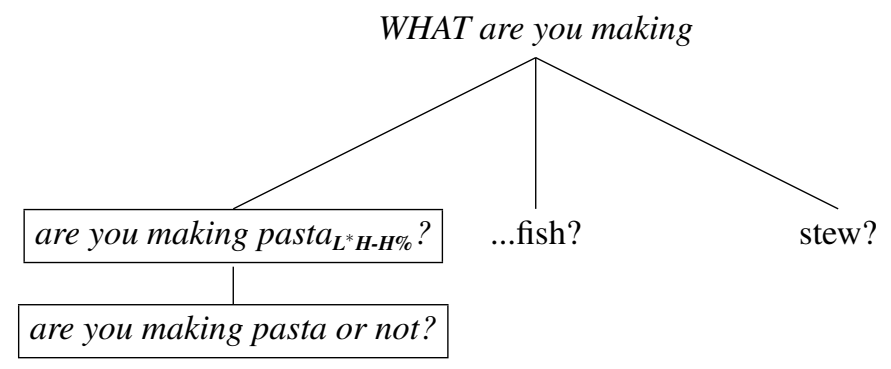

In the dialogue in (38), if the addressee answers no to the ALTQVN, the questioner could then ask whether the addressee is making stew instead (see (39)). This would be a case in which the questioner tries to help the addressee figure out which task he wants to be in charge of by discarding one by one each of the alternatives (evaluating all the alternatives at once seems to be overwhelming for the addressee). The dialogue in (38) illustrates that ALTQVNs can be used as strategies to help the questioner achieve the task of solving the previous question. In Büring's terms, "any sub-tree of a d-tree which is rooted in an interrogative move is a strategy." "The ALTQVN is a move that is ultimately part of a strategy to answer the big question: what are you making ? $^{16}$ When the ALTQVN is accepted, the closure presupposition carried by the question is accepted (or pretended to be accepted for conversational purposes (Stalnaker 2002)). POLQs are different. When a POLQ is uttered, other epistemically possible/relevant alternatives are available.

ALTQVNs can be strategies used to obtain an answer for a PolQ. Is there any strategy that could be used to obtain an answer for an ALTQvN? The answer is no. Alternative questions do not have sisters. This is a consequence of the properties of alternative questions discussed above: alternative questions list all the epistemically possible/relevant alternatives (they exhaustify the "discursive space"). There are not further questions that can be used to narrow down an ALTQVN. The only possible move, if the alternative question is accepted by the addressee, is to give an answer to the AltQvN, i.e., yes or no. AltQvNs are a cul de sac.

It is important to point out that the cornering effect is not only the result of the exhaustivity associated with falling intonation. The nature of the alternatives is important too. Notice that a regular alternative question indicates exhaustivity with

\footnotetext{
${ }^{15}$ This definition follows Roberts (1996). Roberts writes: "[Strategies are] questions designed to (at least) partially satisfy the aims of the game while obeying the game's constraints. Given that the main goal is to answer the Big Question, a reasonable strategy will involve a plan to do this by developing sub-goals which are easier to achieve and are logically related to each other in such a way as to facilitate achieving the main goal."

${ }^{16}$ Notice that the d-tree in (39) is well formed in Büring's terms.
} 
respect to the alternatives available but there is no cornering. This is because other questions can be use as strategies to answer the alternative question, (40).

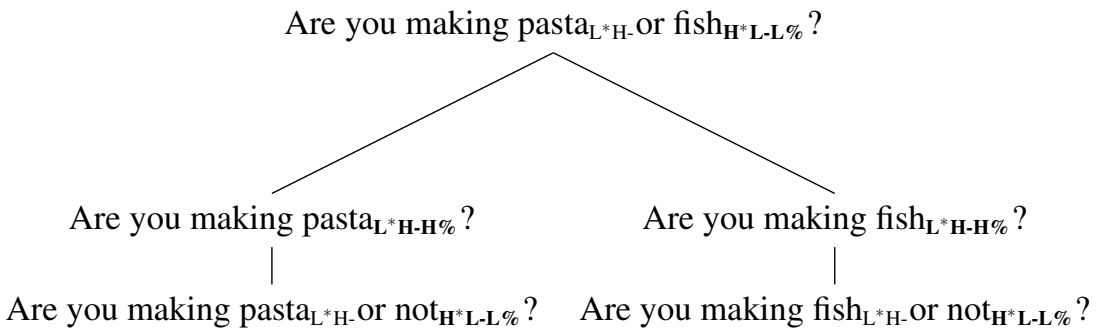

Once the alternative question has been asked, the questioner can go on asking for the different alternatives spelled out (as a strategy to solve the question under discussion). It is when the question asks about opposite alternatives that we find the cornering effect. ALTQvNs are the last moves available to the questioner to try to solve the question under discussion. The issue cannot be narrowed down any further. The only move left to the addressee is to give an answer to the ALTQVN, yes or no. Notice that a sequence of two questions Q1-Q2, in which Q1 is an ALTQVN and Q2 is a PoLQ, is not felicitous, (41).
A: Are you making pasta or not?
B: (silence)

A: \#Are you making pasta?

The AltQvN is the last question about a discourse-topic. At the point the questioner utters an ALTQVN, the discourse is just about making pasta, not about what you want to cook. There is no alternative to making pasta available at that point in the d-tree, since making pasta is the only epistemically possible/relevant alternative. A POLQ cannot be asked after an ALTQVN because once it has been established that there are only two epistemically available alternatives, making pasta or not making pasta, it is not possible to make a move that allows the addressee to think that there may be others, which is what happens with a PoLQ.

The fact that ALTQVNs are the last question in a sequence of questions and that they do not have sisters explains the cornering effect. AltQvNs are the preferred question to close open issues.

\section{Back to Bolinger's data}

Recall that we began by illustrating the differences between POLQs and ALTQvNs with Bolinger's data. Let us now examine how the proposal offered in this paper can explain the cases of requests, invitation and conversation starters, in which the use of a POLQ is fine, whereas the use of an ALTQVN is not discursively appropriate.
a. Will you marry me or not?
[odd]
b. Do you want something to drink or not?
[odd]
c. Do you play golf or not?
[odd] 
We saw that ALTQvNs do not leave the addressee any room to maneuver. The only thing that the addressee can do after the questioner utters an ALTQVN is to give an answer. They are the last move, and this seems to indicate that there has been some previous discussion on the topic. However, in the situations listed above, there is no previous discussion, hence, the utterance of an ALTQVN is odd.

Let us turn now to the cases of inference drawing.
A: I just saw David
B: Is David back from Toronto or not?
[odd]

The use of an ALtQvN question in (43) is odd, whereas a POLQ would be fine. Were B to use a POLQ (is David back from Toronto?), he would only be trying to clarify a secondary issue that came to his mind during the conversation, i.e. whether David got back from Toronto. This is not the case with an ALTQvN. For one, the cornering strategy is justified only if A has "misbehaved" and not been straightforward, and in (43) there is no evidence that A has misbehaved. Second, since after the ALtQvN the only possible move by the addressee is an answer, the use of an ALTQVN 'forces' A to talk about a topic different from the one A seemed to be interested in. This makes the questioner's move odd.

The case of rhetorical questions is similar.

Are you crazy or not? [odd]

Whereas a POLQ like are you crazy? is completely fine as a rhetorical question, an AltQvN is not discursively appropriate. As in (43), the cornering strategy is not justified in the situations in which the question is asked. POLQs are good rhetorical questions since they can be used to clarify a secondary issues: given what has been happening one comes to wonder whether you are crazy. ALTQVNs are not fit for this task. With an ALTQVN the questioner tries to make the issue presented by the question the main topic of the discourse and acts as if there had been some discussion about it. However, in the situations in which a rhetorical question is used, the issue was never open in the first place.

\section{Conclusion}

The central claim of this paper is that the cornering effect is at the core of the difference between POLQs and ALTQvNs. I have argued that the cornering effect is the result of semantic factors and the way those factors shape discourse. Both kinds of questions, POLQs and AltQvNs, involve lists. However, there is a crucial difference in their semantics. In POLQs the list of alternatives is not exhaustive and it is understood that there may be other alternatives available in the context. In the case of ALTQVNs, there is a closure operator in the semantics, signaled by falling intonation, which triggers the exhaustivity presupposition. This difference in the semantics triggers differences in the discourse. Given the fact that ALTQVNs offer opposing alternatives, they become the last possible questioner's move: after an 
AltQvN, the only possible response by a cooperative addressee is an answer to the question. This proposal also accounts for Bolinger's (1978) data.

\section{References}

Alonso-Ovalle, Luis: 2006, Disjunction in Alternative Semantics. GLSA publications, Amherst.

Bolinger, Dwight: 1978, 'YES-NO Questions are not Alternative Questions', in H. Hiz (ed.), Questions, 87 - 105. Reidel Publishing Company, Dordrecht, Holland.

Büring, Daniel: 2003, 'On D-Trees, Beans, and B-Accents', Linguistics \& Philosophy 26, 511-545.

Geurts, Bart: 2005, 'Entertaining Alternatives: Disjunctions as Modals', Natural Language Semantics 13, 383-410.

Groenendijk, Jeroen and Martin Stokhof: 1984, Studies in the Semantics of Questions and the Pragmatics of Answers. University of Amsterdam, Ph.D. Thesis.

Hamblin, Charles Leonard: 1973, 'Disjunction, Focus, and Scope', Foundations of Language 10, 41-53.

Karttunen, Lauri: 1977, 'Syntax and Semantics of Questions', Linguistics \& Philosophy 1, 3-44.

Pruitt, Kathryn: 2007, 'Perceptual Relevance of Prosodic Features in Non-whQuestions with Disjunction'. UMass Amherst.

Pruitt, Kathryn: 2008, 'Prosody and Focus in Alternative Questions: Accounting for Interpretation', in Experimental and Theoretical Advances in Prosody. Cornell, Ithaca, NY.

Roberts, Craige: 1996, 'Information Structure in Discourse: Towards an Integrated Formal Theory of Pragmatics', in OSU Working papers in linguistics 49: papers in semantics, 91-136.

Romero, Maribel and Chung Hye Han: 2003, 'Focus, Ellipsis and the Semantics of Alternative Questions', in P. C. H. F. C. Claire Beyssade, Olivier Bonami (ed.), Empirical Issues in Formal Syntax and Semantics, Vol. 4, 291-307. Presses Universitaires de Paris-Sorbonne, Paris.

van Rooy, Robert and Marie Safárová: 2003, 'On Polar Questions', in R. Young and Y. Zhou (eds.), Semantics and Linguistics Theory 13. Ithaca, NY, Cornell University.

Stalnaker, Robert: 1978, 'Pragmatics', in P. Cole (ed.), Pragmatics Syntax and Semantics. Academic Press, New York.

Stalnaker, Robert: 2002, 'Common Ground', Linguistics and Philosophy 25, 701721.

Zimmermann, Thomas Ed: 2000, 'Free Choice Disjunction and Epistemic Possibility', Natural Language Semantics 8, 255-290. 